\title{
JAMMING COMMUNICATION NETWORKS UNDER COMPLETE UNCERTAINTY
}

\author{
CLAYTON W. COMMANDER, PANOS M. PARDALOS, VALERIY RYABCHENKO, OLEG SHYLO, \\ STAN URYASEV, AND GRIGORIY ZRAZHEVSKY
}

\begin{abstract}
This paper describes a problem of interdicting/jamming wireless communication networks in uncertain environments. Jamming communication networks is an important problem with many applications, but has received relatively little attention in the literature. Most of the work on network interdiction is focused on preventing jamming and analyzing network vulnerabilities. Here, we consider the case where there is no information about the network to be jammed. Thus, the problem is reduced to jamming all points in the area of interest. The optimal solution will determine the locations of the minimum number of jamming devices required to suppress the network. We consider a subproblem which places jamming devices on the nodes of a uniform grid over the area of interest. The objective here is to determine the maximum grid step size. We derive upper and lower bounds for this problem and provide a convergence result. Further, we prove that due to the cumulative effect of the jamming devices, the proposed method produces better solutions than the classical technique of covering the region with uniform circles.
\end{abstract}

\section{INTRODUCTION}

This paper describes a problem of interdicting/jamming communication networks in uncertain environments. Jamming communication networks is an important problem but has not been intensively researched despite the vast amount of work on optimizing telecommunication systems [8]. Most papers on network interdiction are about preventing jamming and analyzing network vulnerability [7,3]. To our knowledge, the only literature on network interdiction involving optimal placement of jamming devices is the work of Commander et al. [1] in which several mathematical programming formulations were given for the deterministic WIRELESS NETWORK JAMMING PROBLEM. The only other thoroughly studied cases are problems of minimizing the maximal network flow and maximizing the shortest path between given nodes via arc interdiction using limited resources. Wood [9], Israeli et al. [5], and Cormican et al. [2] studied stochastic and deterministic cases and suggested efficient heuristics. A similar setup but with a different objective was recently studied by Held in 2005 [4].

Since most situations arise in military battlefield scenarios, exact information about the topology of the adversary's network is unknown. Thus, deterministic network interdiction approaches have limited applicability. In this case, a stochastic approach involving some risk measure for evaluating the efficiency of the jamming device placement may be helpful. However, choosing an appropriate risk measure is a challenging problem in its own right. In this paper, we consider an extreme case where there is no a priori information about the topology of the network to be jammed. The only information used in our approach is a bounding area, containing the communication network.

The organization of the paper is as follows. Section 2 gives a formal description of the problem and the jamming model. We derive bounds and prove a convergence result for the case of complete uncertainty in Section 3. Here we also demonstrate the advantage

Air Force Research Laboratory Technical Report: AAC/PA \#03-29-07-228

To appear in Optimization Letters. 
of the proposed method compared to the simplified case which does not account for the cumulative effect of the jamming devices. Section 4 provides some concluding remarks.

\section{Descriptions, Assumptions, And DeFinitions}

In general, the problem of jamming a communication network is to determine the minimum number of jamming devices required to interdict or suppress functionality of the network. Starting with this general statement, more specific ones can be obtained by considering various types of jamming devices and interdiction criteria. Depending on the given information about the communication nodes and the network topology, stochastic or deterministic setups can be constructed [1]. Below we provide assumptions and basic definitions of the considered framework.

We consider radio-transmitting communication networks and jamming devices operating with electromagnetic waves. We assume that the jamming devices have omnidirectional antennas and emit electromagnetic waves in all directions with the same intensity. We also assume that jamming power decreases reciprocally to the squared distance from a device.

Definition 1. A point (communication node) $X$ is said to be jammed or covered if the cumulative energy received from all jamming devices exceeds some threshold value $E$ :

$$
\sum_{i} \frac{\lambda}{\mathcal{R}^{2}(X, i)} \geq E,
$$

where $\lambda \in \mathbb{R}$ and $\mathcal{R}(X, i)$ represents the distance from $X$ to jamming device $i$. This condition can be rewritten as:

$$
\sum_{i} \frac{1}{\mathcal{R}^{2}(X, i)} \geq \frac{1}{L^{2}}
$$

where $L=\sqrt{\frac{\lambda}{E}}$.

The latter inequality implies that a jamming device covers any point inside a circle of radius $L$.

Definition 2. A connection (arc) between two communication nodes is considered blocked if any of the two nodes is covered.

Usually, interdiction efficiency is determined by a fraction of covered nodes and/or arcs. More complicated criteria used are based on the amount of information transmitted through the network or the length of the shortest path between pairs of nodes. We do not consider a specific criterium because we are interested in the case of complete uncertainty. Thus, we are assuming that we have no knowledge of the network topology, including information about the node coordinates.

\section{JAMming Under COMPlete UnCERTAinty}

If we ignore the cumulative effect of the jamming devices, then the problem reduces to determining the optimal covering of an area on a plane by circles. This covering problem was solved in 1936 by Kershner [6]. The current paper shows that accounting for the cumulative effect of all the devices can lead to significant losses in costs, i.e. required number of jamming devices.

Since we assume no information is known about the network to be jammed, the only reasonable approach is to cover all points in some area known to contain the network. This approach would also be appropriate when some information about the network is available, but is potentially inaccurate.

We consider a case when a communication network is located inside a square. However, all of the following theorems can be formulated for a more general case. For example, to 
obtain results when the network is contained inside a rectangular region in the plane, the only modification required to the calculations is an appropriate updating of the summation bounds.

An optimal covering is one which contains the minimum number of jamming devices that jam all points in the particular area of interest. However, finding a globally optimal solution for the general problem is difficult [1]. Therefore, we consider a subproblem of covering a square with jamming devices located at the nodes of a uniform grid. The solution to this problem will provide a feasible solution (optimal in certain cases) to the general problem. Suppose the grid step size is $R$. If the length of a square side $a$ is not a multiple of $R$, then we cover a bigger square with a side of length $R\left(\left[\frac{a}{R}\right]+1\right)$. See Figure 1 for an example. The optimal solution in the considered problem is a uniform grid with the largest possible step size which covers the square. The problem remains non-trivial, even for this simplified setup.

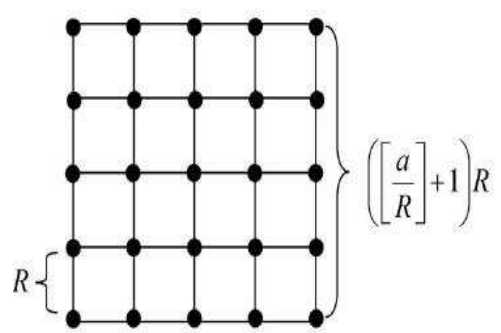

Figure 1: Uniform grid with jamming devices

Lemma 1. For any covering of a square with a uniform grid, a point which receives the least amount of jamming energy lies inside a corner grid cell (see Figure 2).

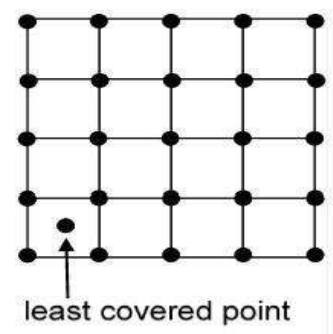

Figure 2: The least covered point is shown in the lower left grid cell.

Proof. Consider a corner cell $S_{0}$ and an arbitrary non-corner cell $S_{i}$. We prove that for any point $P \in S_{i}$, there is a corresponding point $P^{\prime} \in S_{0}$ such that $E(P)>E\left(P^{\prime}\right)$, where $E(X)$ is the cumulative jamming energy from all devices received at point $X$.

Let $P^{\prime}$ be a symmetric correspondence of point $P$ inside $S_{0}$. Here, symmetry implies that $P$ and $P^{\prime}$ are equidistant from the sides of their respective cells. We split the square into the four rectangles $A, B, C$, and $D$, where $A$ is the rectangle containing cells $S_{0}$ and $S_{i}$ (see Figure 3). Denote the other two corner cells of rectangle $A$ by $C_{1}$ and $C_{2}$. Let also $T_{1}$ and $T_{2}$ be points inside $C_{1}$ and $C_{2}$ respectively, such that $T_{1} P T_{2} P^{\prime}$ is a rectangle 


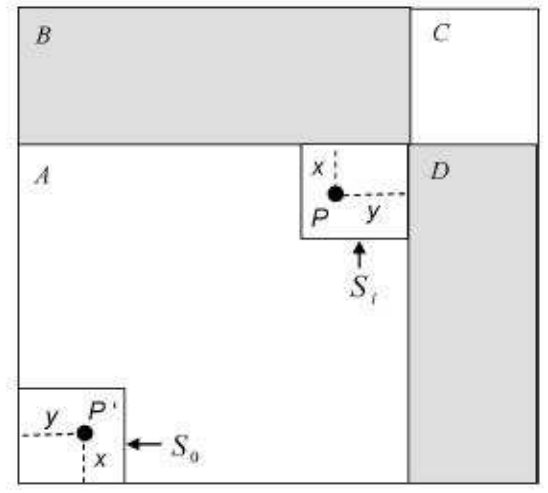

Figure 3: Square Decomposition

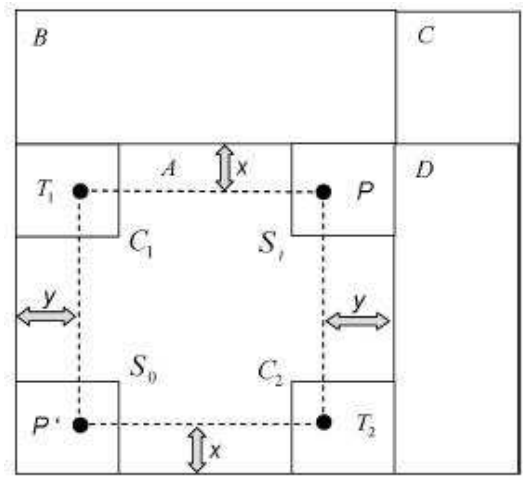

Figure 4: Equivalent Points

with sides parallel to the sides of the square as in Figure 4. Using symmetry we get the following relations:

$$
\begin{aligned}
E\left(P^{\prime}, A\right) & =E(P, A), \\
E\left(P^{\prime}, B\right)<E\left(T_{1}, B\right) & =E(P, B), \\
E\left(P^{\prime}, D\right)<E\left(T_{2}, D\right) & =E(P, D), \\
E\left(P^{\prime}, C\right) & <E(P, C),
\end{aligned}
$$

where $E(X, I)$ is the cumulative jamming energy from all devices inside rectangle $I$ received by point $X$. Relations (3) - (6) imply

$$
\begin{aligned}
E\left(P^{\prime}\right) & =E\left(P^{\prime}, A\right)+E\left(P^{\prime}, B\right)+E\left(P^{\prime}, C\right)+E\left(P^{\prime}, D\right) \\
& <E(P, A)+E(P, B)+E(P, C)+E(P, D) \\
& =E(P),
\end{aligned}
$$

and the lemma is proved.

Below we formulate theorems for upper $\bar{R}$ and lower $\underline{R}$ bounds for the optimal grid step size $R^{*}: \underline{R}<R^{*}<\bar{R}$. In all formulated theorems, we consider covering a square with side length $a$. 
Theorem 1. The unique solution of the equation

$$
\frac{1}{2 R^{2}}\left(\pi \ln \left(\frac{a}{R}+1\right)+\pi-3\right)=\frac{1}{L^{2}}
$$

is a lower bound $\underline{R}$ for the optimal grid step size $R^{*}$.

Proof. In Lemma 1, we proved that the least covered point lies inside a corner cell. Consider now a grid with step size $R$. Without the loss of generality, let $P\left(x_{0}, y_{0}\right)$ be a point

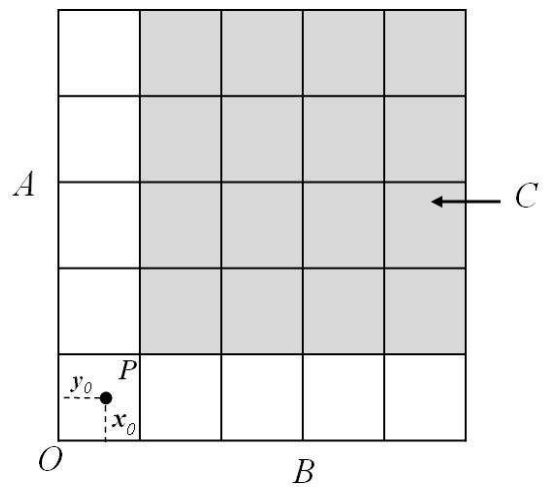

Figure 5: Cumulative emanation of jamming devices.

inside the bottom left corner cell as shown in Figure 5. $I_{1}, I_{2}$, and $I_{3}$ are cumulative jamming energy received at $P$ by jamming devices located in regions $C, A$, and $B$ correspondingly. Similarly, $I_{4}$ is the jamming energy from the jamming device located at the bottom left node $O$. With this, the jamming energy received at point $P$ is calculated through the expression

$$
\begin{array}{r}
E(P)=I_{1}+I_{2}+I_{3}+I_{4}, \text { where } \\
I_{1}=\sum_{i=0}^{T-1} \sum_{j=0}^{T-1} \frac{1}{\left(R-x_{0}+i \cdot R\right)^{2}+\left(R-y_{0}+j \cdot R\right)^{2}}, \\
I_{2}=\sum_{i=0}^{T-1} \frac{1}{\left(R-x_{0}+i \cdot R\right)^{2}+y_{0}^{2}}, \\
I_{3}=\sum_{j=0}^{T-1} \frac{1}{x_{0}^{2}+\left(R-y_{0}+j \cdot R\right)^{2}}, \\
I_{4}=\frac{1}{x_{0}^{2}+y_{0}^{2}}, \\
T=\left[\frac{a}{R}\right]+1 .
\end{array}
$$

Notice that we can estimate $I_{2}+I_{3}$ as

$$
I_{2}+I_{3} \geq 2 \cdot \sum_{i=0}^{T-1} \frac{1}{R^{2}(1+i)^{2}+R^{2}} \geq \frac{2}{R^{2}} \int_{0}^{T} \frac{1}{1+(1+x)^{2}} d x .
$$

This follows from the fact that

$$
\sum_{i=0}^{N} f(i) \geq \int_{0}^{N+1} f(x) d x
$$




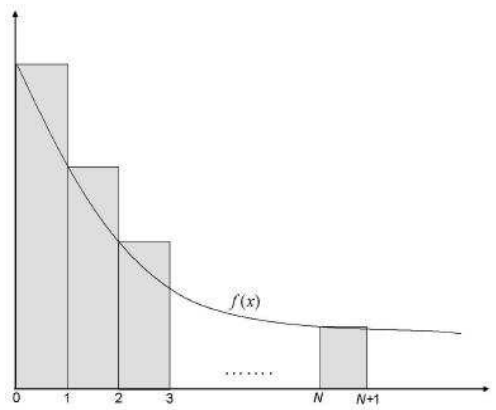

Figure 6: Integral Lower Bound.

where $f(x)$ is a decreasing function. This property can be easily established geometrically. Notice in Figure 6 that the left side of inequality (16) represents the shaded region in the figure, while the right side represents the area under $f(x)$. Continuing from (15) above we have

$$
\begin{aligned}
\int_{0}^{T} \frac{1}{1+(1+x)^{2}} d x & =\arctan (T+1)-\frac{\pi}{4} \\
& =\frac{\pi}{2}-\arctan \left(\frac{1}{T+1}\right)-\frac{\pi}{4} \\
& \geq \frac{\pi}{4}-\frac{1}{T+1} .
\end{aligned}
$$

Here and further, we use the inequalities given below:

$$
\begin{array}{r}
\arctan (x) \leq x, 0 \leq x \leq 1, \\
\arctan (x) \geq x-\frac{x^{3}}{3}, 0 \leq x \leq 1 .
\end{array}
$$

Now combining (15) and (17), we obtain

$$
I_{2}+I_{3} \geq \frac{2}{R^{2}}\left(\frac{\pi}{4}-\frac{1}{T+1}\right)
$$

We also have the following approximation for $I_{4}$ which follows clearly

$$
I_{4} \geq \frac{1}{2 R^{2}} \text {. }
$$

For estimating $I_{1}$ we use a property similar to (16), but in a higher dimension. Namely,

$$
\sum_{i=0}^{N} \sum_{j=0}^{N} f(i, j) \geq \int_{0}^{N+1} \int_{0}^{N+1} f(x, y) d x d y
$$

where as above, $f(x, y)$ is a decreasing function of $x$ and $y$. Using this inequality, we derive the following approximation for $I_{1}$.

$$
\begin{aligned}
I_{1} & \geq \int_{0}^{T} \int_{0}^{T} \frac{d x d y}{\left(R-x_{0}+x \cdot R\right)^{2}+\left(R-y_{0}+y \cdot R\right)^{2}} \\
& \geq \int_{0}^{T} \int_{0}^{T} \frac{d x d y}{(R+x \cdot R)^{2}+(R+y \cdot R)^{2}} \\
& =\frac{1}{R^{2}} \int_{1}^{T+1} \int_{1}^{T+1} \frac{d x d y}{x^{2}+y^{2}} .
\end{aligned}
$$


Furthermore,

$$
\begin{aligned}
\int_{1}^{T+1} \int_{1}^{T+1} \frac{d x d y}{x^{2}+y^{2}} & =\int_{1}^{T+1} \frac{1}{x} \arctan \left(\frac{T+1}{x}\right) d x-\int_{1}^{T+1} \frac{1}{x} \arctan \left(\frac{1}{x}\right) d x \\
& \geq \int_{1}^{T+1} \frac{1}{x} \arctan \left(\frac{T+1}{x}\right) d x-\int_{1}^{T+1} \frac{d x}{x^{2}} \\
& =\int_{1}^{T+1} \frac{1}{x}\left(\frac{\pi}{x}-\arctan \left(\frac{x}{T+1}\right)\right) d x-1+\frac{1}{T+1} \\
& =\frac{\pi}{2} \ln (T+1)-1+\frac{1}{T+1}-\int_{0}^{T+1} \frac{1}{x} \arctan \left(\frac{x}{T+1}\right) d x \\
& \geq \frac{\pi}{2} \ln (T+1)-1+\frac{1}{T+1}-\int_{0}^{T+1} \frac{1}{x}\left(\frac{x}{T+1}\right) d x \\
& =\frac{\pi}{2} \ln (T+1)-2\left(1-\frac{1}{T+1}\right)
\end{aligned}
$$

Combining this result with (23) we have

$$
I_{1} \geq \frac{1}{R^{2}}\left(\frac{\pi}{2} \ln (T+1)-2\left(1-\frac{1}{T+1}\right)\right) .
$$

Summing (20), (21), and (25) we obtain an overestimate of the total coverage at point $P$. That is

$$
\begin{aligned}
E(P) & \geq \frac{1}{R^{2}} \cdot\left(\frac{\pi}{2} \ln (T+1)-2+\frac{2}{T+1}+\frac{\pi}{2}-\frac{2}{T+1}+\frac{1}{2}\right) \\
& =\frac{1}{R^{2}}\left(\frac{\pi}{2} \ln (T+1)+\frac{\pi}{2}-\frac{3}{2}\right) \\
& \geq \frac{1}{2 R^{2}}\left(\pi \cdot \ln \left(\frac{a}{R}+1\right)+\pi-3\right) .
\end{aligned}
$$

To guarantee coverage of point $P$, it is sufficient to claim that

$$
f(R)=\frac{1}{2 R^{2}}\left(\pi \cdot \ln \left(\frac{a}{R}+1\right)+\pi-3\right) \geq \frac{1}{L^{2}} .
$$

Since $f(R)$ is monotonically decreasing on $(0,+\infty)$, the largest $R$ satisfying the above inequality is the unique solution $\underline{R}$ of the equation

$$
f(R)=\frac{1}{L^{2}}
$$

Thus, a uniform grid with step size $\underline{R}$ jams any point $P$ inside a corner cell. According to Lemma 1, the grid jams the least covered point in the square implying that the whole square is jammed. Thus we have the desired result.

Since the function $f(R)=\frac{1}{2 R^{2}}\left(\pi \ln \left(\frac{a}{R}+1\right)+\pi-3\right)$ is monotonic, equation (8) can be easily solved using a numerical procedure such as a binary search. Therefore, using (8), we can obtain a step size $\underline{R}$ such that the corresponding uniform grid covers the entire square. Further, the number of jamming devices in the grid does not exceed

$$
N_{1}=\left(\frac{a}{\underline{R}}+2\right)^{2} .
$$

A more straightforward solution of the initial problem could be based on the property that a jamming device covers all the points inside a circle of radius $L$ as mentioned in Definition 1. Using that, we could reduce the problem to finding the optimal covering of a square 
with circles of radius $L$. A direct result from [6] (that was mentioned in [7]) is that in the limit, the minimum number of circles to cover an area $a^{2}$ is

$$
N_{2}=\frac{2 a^{2}}{3 \sqrt{3 L^{2}}}
$$

To compare the approaches, we consider the ratio

$$
\begin{aligned}
\frac{N_{2}}{N_{1}} & =\left(\frac{\underline{R}}{L^{2}}\right) \frac{2}{3 \sqrt{3}} \frac{1}{\left(1+2 \frac{\underline{R}}{a}\right)^{2}} \\
& =\frac{2 x^{2}}{3 \sqrt{3}} \frac{1}{\left(1+\frac{2 x}{k}\right)^{2}}
\end{aligned}
$$

where $x=\frac{R}{L}$ and $k=\frac{a}{L}$. Using these substitutions, equation (8) can be rewritten in terms of variables $x$ and $k$ as follows

$$
\frac{1}{x^{2}}\left(\pi \ln \left(\frac{k}{x}+1\right)+\pi-3\right)=2
$$

By solving (32) for different values of $k$, one can find corresponding values of $x$ and $\frac{N_{2}}{N_{1}}$. To evaluate the advantage of the uniform grid approach over the naive one, we provide some computational results in the Table 1 . From the table, we see that as $k$ increases, the

\begin{tabular}{|c|c|c|}
\hline$k$ & $x$ & $\frac{N_{2}}{N_{1}}$ \\
\hline $10^{2}$ & 2.44 & 2.3 \\
\hline $10^{4}$ & 3.54 & 4.8 \\
\hline $10^{6}$ & 4.40 & 7.5 \\
\hline $10^{8}$ & 5.14 & 10.2 \\
\hline
\end{tabular}

Table 1: Comparing $\frac{N_{2}}{N_{1}}$ for various values of $k$.

advantage of using our approach becomes more significant. In fact, it can be proved that $\lim _{a \rightarrow \infty} \frac{N_{2}}{N_{1}}=\infty$. This will follow as a corollary of Theorem 3 .

To establish the quality of the lower bound rigorously, we need to first establish a similar result for an upper bound. This follows in the next theorem.

Theorem 2. The unique solution of the equation

$$
\frac{1}{R^{2}}\left(\frac{\pi}{2} \ln \left(\frac{2 a}{R}+1\right)-\frac{1}{6\left(\frac{a}{R}+1\right)}+\frac{\pi}{2}+\frac{19}{3}\right)=\frac{1}{L^{2}}
$$

is an upper bound $\bar{R}$ of the optimal grid step size $R^{*}$.

Proof. Let $P\left(x_{0}, y_{0}\right)$ be the least jammed point, that lies inside a corner cell according to Lemma 1. Without the loss of generality, as in the proof of Theorem 1, we assume that $P$ is inside the bottom left corner cell. The jamming energy received at point $P$ is calculated through the expressions (9) - (14). Since $P$ is the least covered point, the following inequality holds. 


$$
\begin{array}{r}
E(P) \leq E\left(P^{\prime}\left(x=\frac{R}{2}, y=0\right)\right)=I_{1}^{\prime}+I_{2}^{\prime}+I_{3}^{\prime}+I_{4}^{\prime}, \text { where } \\
I_{1}^{\prime}=\sum_{i=0}^{T-1} \sum_{j=0}^{T-1} \frac{1}{\left(\frac{R}{2}+i \cdot R\right)^{2}+(R+j \cdot R)^{2}}, \\
I_{2}^{\prime}=\sum_{i=0}^{T-1} \frac{1}{\left(\frac{R}{2}+i \cdot R\right)^{2}}, \\
I_{3}^{\prime}=\sum_{j=0}^{T-1} \frac{1}{\left(\frac{R}{2}\right)^{2}+(R+j \cdot R)^{2}}, \\
I_{4}^{\prime}=\frac{1}{\left(\frac{R}{2}\right)^{2}} .
\end{array}
$$

$I_{2}^{\prime}$ and $I_{3}^{\prime}$ can be estimated through integrals similarly to the techniques used in the proof of Theorem 1 . The following inequality holds

$$
\sum_{i=1}^{N} f(i) \leq \int_{0}^{N} f(x) d x
$$

where $f(x)$ is a decreasing function. This property can also be proven geometrically. Figure 7 represents a graphical interpretation of this relation. The left side of the inequality

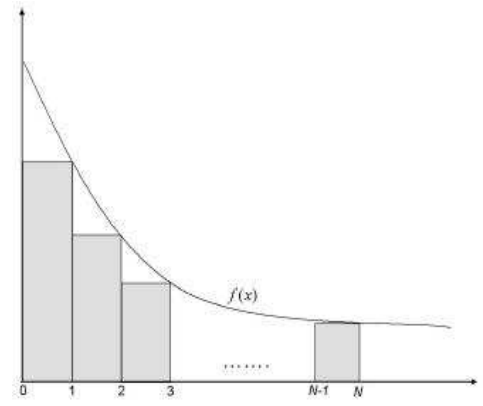

Figure 7: Integral Upper Bound.

is represented by the shaded area. The right side of (39) is the area under $f(x)$. With this property we have from (36) that

$$
\begin{aligned}
I_{2}^{\prime} & \leq \frac{1}{\left(\frac{R}{2}\right)^{2}}+\int_{0}^{T-1} \frac{d x}{\left(\frac{R}{2}+x \cdot R\right)^{2}} \\
& =\frac{1}{R^{2}}\left(6-\frac{1}{T-\frac{1}{2}}\right) .
\end{aligned}
$$


Furthermore, using inequalities (18) and (19), we see that (37) is estimated by

$$
\begin{aligned}
I_{3}^{\prime} & \leq \frac{1}{\left(\frac{R}{2}\right)^{2}+(R+x \cdot R)^{2}} \\
& =\frac{2}{3 R^{2}}+\frac{2}{R^{2}}\left(\arctan \left(\frac{1}{2}\right)-\arctan \left(\frac{1}{2 T}\right)\right) \\
& \leq \frac{2}{3 R^{2}}+\frac{2}{R^{2}}\left(\frac{1}{2}-\frac{1}{2 T}+\frac{1}{24 T^{3}}\right) \\
& =\frac{1}{R^{2}}\left(\frac{5}{3}-\frac{1}{T}+\frac{1}{12 T^{3}}\right) .
\end{aligned}
$$

To estimate $I_{1}^{\prime}$ a property similar to (39) can be used. This inequality is given by

$$
\sum_{i=1}^{N} \sum_{j=1}^{N} f(i, j) \leq \int_{0}^{N} \int_{0}^{N} f(x, y) d x d y+\int_{0}^{N} f(x, 0) d x+\int_{0}^{N} f(0, y) d y,
$$

where $f(x, y)$ is a decreasing function of $x$ and $y$. With the above inequality,

$$
\begin{gathered}
I_{1}^{\prime} \leq \frac{1}{\left(\frac{R^{2}}{2}\right)+R^{2}}+\int_{0}^{T-1} \frac{d x}{\left(\frac{R}{2}\right)^{2}+(R+x \cdot R)^{2}}+\int_{0}^{T-1} \frac{d x}{\left(\frac{R}{2}+x \cdot R\right)^{2}+R^{2}}+ \\
+\int_{0}^{T-1} \int_{0}^{T-1} \frac{d x d y}{\left(\frac{R}{2}+x \cdot R\right)^{2}+\left((R+y \cdot R)^{2}\right.} \\
=\frac{4}{5 R^{2}}+\frac{C}{R^{2}}+\frac{1}{R^{2}} \int_{0}^{T-1} \int_{0}^{T-1} \frac{d\left(x+\frac{1}{2}\right) d y}{\left(\frac{1}{2}+x\right)^{2}+(y+1)^{2}}, \text { where } \\
C=2 \arctan (2 T)-\arctan (2)+\arctan \left(T-\frac{1}{2}\right)-\frac{\pi}{2} \\
\quad=\frac{\pi}{2}-2 \arctan \left(\frac{1}{2 T}\right)+\arctan \left(\frac{1}{2}\right)-\arctan \left(\frac{2}{2 T-1}\right) \\
\leq \frac{\pi}{2}-2\left(\frac{1}{2 T}-\frac{1}{24 T^{3}}\right)+\frac{1}{2}-\left(\frac{2}{2 T-1}-\frac{8}{3(2 T-1)^{3}}\right) \\
\leq \frac{\pi+1}{2} .
\end{gathered}
$$

The double integral in (43) is bounded as follows

$$
\begin{aligned}
& \int_{0}^{T-1} \int_{0}^{T-1} \frac{d\left(x+\frac{1}{2}\right) d y}{\left(\frac{1}{2}+x\right)^{2}+(y+1)^{2}}=\int_{\frac{1}{2}}^{T-\frac{1}{2}} \int_{1}^{T} \frac{d t d y}{t^{2}+y^{2}} \\
& =\int_{\frac{1}{2}}^{T-\frac{1}{2}} \frac{1}{t}\left(\arctan \left(\frac{T}{t}\right)-\arctan \left(\frac{1}{t}\right)\right) d t \\
& \leq \int_{\frac{1}{2}}^{T-\frac{1}{2}} \frac{1}{t}\left(\frac{\pi}{2}-\arctan \left(\frac{t}{T}\right)\right) d t-\int_{\frac{1}{2}}^{T-\frac{1}{2}} \frac{1}{t}\left(\frac{1}{t}-\frac{1}{3 t^{3}}\right) d t \\
& \leq \frac{\pi}{2}\left(\ln \left(T-\frac{1}{2}\right)-\ln \left(\frac{1}{2}\right)\right)-\int_{\frac{1}{2}}^{T-\frac{1}{2}} \frac{1}{t}\left(\frac{t}{T}-\frac{t^{3}}{3 T^{3}}\right) d t- \\
& -\left(\frac{4}{3}-\frac{1}{T-\frac{1}{2}}+\frac{1}{6\left(T-\frac{1}{2}\right)^{2}}\right) \\
& =\frac{\pi}{2} \ln (2 T-1)-\frac{20}{3}+\frac{5}{6 T}+\frac{1}{12 T^{2}}-\frac{1}{36 T^{3}}+\frac{1}{T-\frac{1}{2}}-\frac{1}{6\left(T-\frac{1}{2}\right)^{2}} \\
& <\frac{\pi}{2} \ln (2 T-1)-\frac{20}{3}+\frac{5}{6 T}+\frac{1}{T-\frac{1}{2}}-\frac{1}{12\left(T-\frac{1}{2}\right)^{2}} .
\end{aligned}
$$


Combining the results from (43), (44), and (45) gives the overestimate for $I_{1}^{\prime}$ as

$$
I_{1}^{\prime}<\frac{1}{R^{2}}\left(\frac{\pi}{2} \ln (2 T-1)+\frac{\pi}{2}-\frac{16}{3}+\frac{5}{6 T}+\frac{1}{T-\frac{1}{2}}-\frac{1}{12\left(T-\frac{1}{2}\right)^{2}}\right) .
$$

Recall equation (34) stated $E(P) \leq I_{1}^{\prime}+I_{2}^{\prime}+I_{3}^{\prime}+I_{4}$. So using the expression for $I_{4}^{\prime}$ given in (38) and the overestimates for $I_{1}^{\prime}, I_{2}^{\prime}$, and $I_{3}^{\prime}$ derived in equations (46), (40), and (41) respectively, we obtain

$$
E(P) \leq \frac{1}{R^{2}}\left(\frac{\pi}{2} \ln (2 T-1)-\frac{1}{6 T}+\frac{\pi}{2}+\frac{19}{3}\right)
$$

Finally, if we let $T=\left[\frac{a}{R}\right]+1 \leq \frac{a}{R}+1$, we get

$$
E(P)<\frac{1}{R^{2}}\left(\frac{\pi}{2} \ln \left(\frac{2 a}{R}+1\right)-\frac{1}{6\left(\frac{a}{R}+1\right)}+\frac{\pi}{2}+\frac{19}{3}\right)
$$

The function $f(R)=\frac{1}{R^{2}}\left(\frac{\pi}{2} \ln \left(\frac{2 a}{R}+1\right)-\frac{1}{6\left(\frac{a}{R}+1\right)}+\frac{\pi}{2}+\frac{19}{3}\right)$ is monotone, hence the equation $f(R)=\frac{1}{L^{2}}$ has a unique solution $\bar{R}$. Equation (48) implies that a grid with step size $\bar{R}$ does not cover the entire square. That is, there exists at least one point $P$ that remains uncovered. Thus $\bar{R}$ is an upper bound for the optimal grid covering problem. Since the optimal grid step size $R^{*}<\bar{R}$, the theorem is proved.

In Figure 8, we see an example in which we are covering at $40 \times 40$ square and the required jamming level at each point is 3.0 units. In part (a), we see the coverage associated with the required number of devices from the lower bound of Theorem 2 . In this case, $20^{2}=400$ jamming devices are used to cover the area. Notice that there are no holes in the region. This, together with the scallop shell outside the bounding box indicates that all points within the region are covered. In part (b), we see the coverage corresponding to the placement of the jamming devices on a uniform grid according to the upper bound of Theorem 3. Here, the required number of devices is $19^{2}=361$. Notice the holes located at the four corners of the region indicating that these points are uncovered. This validates the theoretical results obtained in Theorem 2 and Theorem 3.

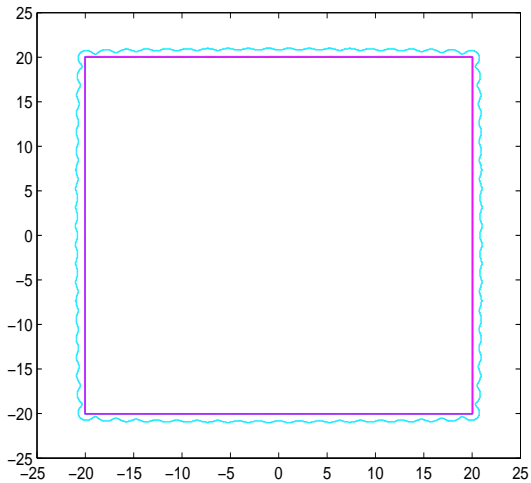

(a)

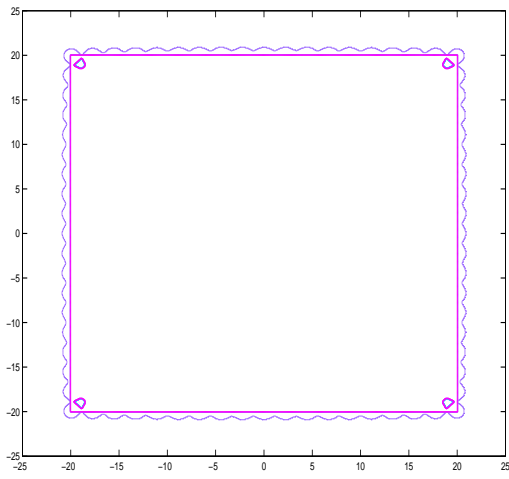

(b)

Figure 8: (a) The coverage when jamming devices are placed according to the lower bound from Theorem 2. The total number of jamming devices required is $20^{2}=400$. (b) We see the coverage associated with the result obtained from Theorem 3 . In this case, $19^{2}=361$ devices are placed. Notice the corner points are not jammed. 
Now that we have established both upper and lower bounds for an optimal grid step size, we can determine the quality of the bounds. The result is obtained in the following theorem.

\section{Theorem 3.}

$$
\lim _{a \rightarrow \infty} \frac{\bar{R}}{\underline{R}}=1,
$$

where $\bar{R}$ and $\underline{R}$ are bounds obtained from equations (8) and (33), correspondingly. Moreover, the following inequality holds:

$$
1 \leq \frac{\bar{R}}{\underline{R}} \leq \sqrt{1+\frac{c}{\ln (a)}},
$$

for constants $M \in \mathbb{R}, c \in \mathbb{R}$, such that $\bar{R}>M$.

Proof. By letting $x=\frac{R}{L}$ and $y=\frac{\bar{R}}{L}$, equations (8) and (33) can be respectively rewritten as

$$
\begin{gathered}
a=L \cdot x\left(e^{\frac{2}{\pi}\left(x^{2}+\frac{3}{2}\right)-1}-1\right), \text { and } \\
\frac{\pi}{2} \ln \left(\frac{2 a}{L \cdot y}+1\right)=y^{2}-\frac{19}{3}-\frac{\pi}{2}+\frac{L \cdot y}{6(a+L \cdot y)} .
\end{gathered}
$$

To prove the theorem, we need to show that

$$
\lim _{a \rightarrow \infty} \frac{y}{x}=1,
$$

where $x>0$ and $y>0$ are solutions of (51) and (52), correspondingly. From (52), we obtain

$$
\begin{array}{r}
\frac{\pi}{2} \ln \left(\frac{2 a}{L \cdot y}+1\right)>y^{2}-C_{1}, \text { where } \\
C_{1}=\frac{19}{3}+\frac{\pi}{2}, \text { and } \\
a>\frac{L \cdot y}{2}\left(e^{\frac{2}{\pi}\left(y^{2}-C_{1}\right)}-1\right) .
\end{array}
$$

From (51) and (56) we see that

$$
\begin{array}{r}
x\left(e^{\frac{2}{\pi}\left(x^{2}+C_{2}\right)} \cdot C_{3}-1\right)>\frac{y}{2}\left(e^{\frac{2}{\pi}\left(y^{2}-C_{1}\right)}-1\right), \text { where } \\
C_{2}=\frac{3}{2}, \text { and } \\
C_{3}=e^{-1} .
\end{array}
$$

Since $y \cdot L$ and $x \cdot L$ are upper and lower bounds, correspondingly, the following relation holds

$$
\frac{y}{x}>1 .
$$

With (51) and (60) above, we can also conclude that

$$
\lim _{a \rightarrow \infty} x=\infty \text { and } \quad \lim _{a \rightarrow \infty} y=\infty .
$$

For all $M \in \mathbb{R}$, where $M>\sqrt{C_{1}}$, there exists $Q \in \mathbb{R}$ such that (57) can be reduced to

$$
\frac{y}{x}<Q \cdot e^{\frac{2}{\pi}\left(x^{2}-y^{2}\right)} \text {, and } y>M .
$$

Moreover, for $c=\frac{\pi}{2} \ln (Q)$ the following inequality holds

$$
\left(\frac{y}{x}\right)^{2}-1 \leq \frac{c}{x^{2}} \text {, and } y>M .
$$


Assume for the sake of contradiction that the inequality in (63) does not hold for some $\left(x^{*}, y^{*}\right)$. That is assume that $\left(\frac{y^{*}}{x^{*}}\right)^{2}-1>\frac{c}{x^{* 2}}$. Using (62) we have

$$
\frac{y^{*}}{x^{*}}<Q \cdot e^{-\frac{2}{\pi} x^{* 2}\left(\left(\frac{y^{*}}{x^{*}}\right)^{2}-1\right)}<Q \cdot e^{-\frac{2}{\pi} x^{* 2} \cdot \frac{c}{x^{* 2}}}=1
$$

which contradicts (60).

Applying (60) and (63) we get

$$
1<\frac{y}{x} \leq \sqrt{1+\frac{c}{x^{2}}}, \text { and } y>M .
$$

Letting $a$ tend to $\infty$ and taking (61) into account, we see that in fact

$$
\lim _{a \rightarrow \infty} \frac{y}{x}=1 \text {. }
$$

Finally, by using (65) and (51), the following relation can be obtained

$$
1<\frac{y}{x} \leq \sqrt{1+\frac{k}{\ln (a)}},
$$

for some constant $k \in \mathbb{R}$, when $y>M$. Thus, the theorem is proved.

\section{CONCLUSiOn}

In this paper, we introduced the problem of jamming a communication network under complete uncertainty. We examined the case when the network is known to lie in a square with area $a^{2}$. We derived upper and lower bounds for the optimal number of jamming devices required when they are located at the vertices of a uniform grid. We also provided a convergence result indicating that the proposed bounds are tight. Furthermore, we proved that our approach is more efficient than the solution provided by optimally covering the square with circles of radius $L$.

\section{ACKNOWLEDGEMENTS}

The authors gratefully acknowledge the Air Force Office of Scientific Research for providing funding under project: FA-9550-05-1-0137.

\section{REFERENCES}

[1] C.W. Commander, P.M. Pardalos, V. Ryabchenko, and S. Uryasev. The wireless network jamming problem. Journal of Combinatorial Optimization, submitted, 2006.

[2] K.J. Cormican, D.P. Morton, and R.K. Wood. Stochastic network interdiction. Operations Research, 46(2):184-197, 1998.

[3] A. Farago. Graph theoretic analysis of ad hoc network vulnerability. In Modeling and Optimization in Mobile, Ad Hoc and Wireless Networks (WiOpt '03), 2003.

[4] H. Held, R. Hemmecke, and D.L. Woodruff. A decomposition algorithm applied to planning the interdiction of stochastic networks. Naval Research Logistics, 52(4):321-329, 2005.

[5] E. Israeli and R.K. Wood. Shortest-path network interdiction. Networks, 40(2):97-111, 2002.

[6] R. Kershner. The number of circles covering a set. American Journal of Mathematics, 61(3):665-671, 1939.

[7] G. Noubir. On connectivity in ad hoc networks under jamming using directional antennas and mobility, volume 2957 of Lecture Notes in Computer Science, pages 186-200. Springer, 2004.

[8] M.G.C. Resende and P.M. Pardalos. Handbook of Optimization in Telecommunications. Springer, 2006.

[9] K. Wood. Deterministic network interdiction. Mathematical and Computer Modeling, 17(2):1-18, 1993.

(C.W. Commander) Air Force Research Laboratory, Munitions Directorate, and, Dept. of Industrial AND Systems ENGINEERING, University of FLORIDA, GAINESVILle, FL USA.

E-mail address: clayton. commander@eglin.af.mil

(P.M. Pardalos and O. Shylo) Center for Applied Optimization, Dept. of Industrial and Systems ENGINEERING, UNIVERSiTy OF FloRidA, GAINESVILle, FL, USA.

E-mail address: $\{$ pardalos, shylo\}@ufl.edu 
(V. Ryabchenko S. Uryasev, and G. ZraZhevsky) Risk Management and Financial EngiNEERING LAB, DEPT. OF INDUSTRIAL AND SYSTEMS ENGINEERING, UNIVERSITY OF FLORIDA, GAINESVILLE, FL USA.

E-mail address: $\{$ valeriy, uryasev\}@ufl.edu 